SU-ITP-04/08

SLAC-PUB-10226

March, 2004

hep-th/yymmnnn

\title{
Leptogenesis from Gravity Waves in Models of Inflation
}

\author{
Stephon H. S. Alexander and Michael E. Peskin ${ }^{1}$ \\ Stanford Linear Accelerator Center, Stanford University \\ 2575 Sand Hill Rd., Menlo Park, CA 94025 USA \\ and \\ M. M. SHEIKH-JABBARI ${ }^{2}$ \\ Institute for Theoretical Physics, Dept. of Physics, Stanford University \\ 382 via Pueblo Mall, Stanford CA 94305-4060 USA
}

\begin{abstract}
We present a new mechanism for creating the observed cosmic matterantimatter asymmetry which satisfies all three Sakharov conditions from one common thread, gravitational waves. We generate lepton number through the gravitational anomaly in the lepton number current. The source term comes from elliptically polarizated gravity waves that are produced during inflation if the inflaton field contains a CP-odd component. In simple inflationary scenarios, the generated matter asymmetry is very small. We describe some special conditions in which our mechanism can give a matter asymmetry of realistic size.
\end{abstract}

\footnotetext{
${ }^{1}$ Work supported by the US Department of Energy, contract DE-AC03-76SF00515.

${ }^{2}$ Work supported in part by the US National Science Foundation, contract PHY-9870115.
} 


\section{Introduction}

As far into the universe as we can see, there is an excess of matter over anti-matter. The recent determinations of the cosmological parameters from the cosmic microwave background by the WMAP experiment gives the baryon density of the universe as [1]

$$
\frac{n_{b}}{n_{\gamma}}=(6.5 \pm 0.4) \times 10^{-10}
$$

This is a small number, but at the same time it is large enough to be a puzzle for models of particle physics. A baryon excess this large cannot be produced in the early universe within the Standard Model of particle physics [2]. Models that can explain the baryon excess typically involve exotic nonstandard physics, $\mathrm{CP}$ violating couplings in the Higgs or supersymmetry sectors or in the couplings of the heavy neutral leptons associated with neutrino mass [3]. In this paper, we introduce a new mechanism for the creation of the matter-antimatter asymmetry, one associated with gravitational fluctuations created during cosmological inflation.

The conditions for generating a matter-antimatter asymmetry were stated by Sakharov almost forty years ago [4]. First, baryon number should be violated. Second, CP should be violated. Third, these symmetry violations should be relevant at a time when the universe is out of thermal equilibrium. Since the 1980's, it has been realized that the standard weak interactions contain processes, mediated by sphaelerons, which interconvert baryons and leptons and are thermally activated at temperatures greater than $1 \mathrm{TeV}$. Thus, we can also create the baryon asymmetry by creating net lepton number at high temperature through out-of-equilibrium and CP-asymmetric processes $[5,6]$. Scenarios of this type are known as leptogenesis.

The out-of-equilibrium conditions can be created at a phase transition or through late decay of massive particles. The most attractive choice for a phase transition is that associated with electroweak symmetry breaking. However, that phase transition is probably not sufficiently strongly first-order. This is known to be an obstacle to baryogenesis in the Standard Model, and most of the allowed parameter space in minimal supersymmetry is also already excluded. Particle decay asymmetries are loop-suppressed and therefore require relatively large CP-violating phases. Such large phases are strongly constrained in supersymmetry [7] though they still could appear in the neutrino Yukawa couplings that are used in the Fukugita-Yanagida scenario for leptogenesis [6]. In any event, there is good reason to seek more effective sources of CP-violating out-of-equilibrium physics. 


\section{Outline of the mechanism}

We would like to assemble a theory of matter-antimatter asymmetry out of the following ingredients. First, as is well-known [8], the lepton number current, and also the total fermion number current, has a gravitational anomaly in the Standard Model. Explicitly,

$$
\partial_{\mu} J_{\ell}^{\mu}=\frac{1}{16 \pi^{2}} R \tilde{R}
$$

where

$$
J_{\ell}^{\mu}=\bar{\ell}_{i} \gamma^{\mu} \ell_{i}+\bar{\nu}_{i} \gamma^{\mu} \nu_{i}, \quad R \tilde{R}=\frac{1}{2} \epsilon^{\alpha \beta \gamma \delta} R_{\alpha \beta \rho \sigma} R_{\gamma \delta}{ }^{\rho \sigma} .
$$

The anomaly requires an imbalance of left- and right-handed leptons, so we are ignoring right-handed neutrinos. In general, (2) will be correct in an effective theory valid below a scale $\mu$. A simple guess for $\mu$ is that it is at the right-handed neutrino scale, of the order of $10^{14} \mathrm{GeV}$, but we would like to keep in mind the possibility of higher values of $\mu$.

Next, we claim, a contribution to $R \tilde{R}$ of definite sign can be generated by gravitational fluctuations produced during inflation if the inflaton field contains a component of odd CP. This can be naturally achieved if the inflaton is a complex modulus field such as one finds in supergravity or superstring models. Such fields can have the very flat potentials required for inflation. The imaginary part $\phi$ of this field (which we henceforth call an 'axion') can couple to gravity through an interaction

$$
\Delta \mathcal{L}=F(\phi) R \tilde{R}
$$

where $F$ is odd in $\phi$, as a result of the Green-Schwarz mechanism [9]. Lue, Wang, and Kamionkowski (LWK) have studied the effects of such an interaction in generating observable parity-violation in the cosmic microwave background [10]. A simple form for $F(\phi)$ with the correct scaling is

$$
F(\phi)=\frac{1}{16 \pi^{2} M_{\mathrm{Pl}}} \mathcal{N} \phi
$$

where the $M_{\mathrm{Pl}}$ in the denominator is approximately the string scale. In principle, we could imagine substituting a lower mass scale $\mathcal{F}$ in the denominator of $(5)$, with the constraint that our effective field theory is valid only for $\mu<\mathcal{F}$.

We would like to apply the interaction (4) to the dynamics of metric fluctuations during inflation. When the axion field has a slowly-rolling nonzero classical value, the coupling (4) can lead to quantum fluctuations of the gravitational field that, treated to second order, generate a nonzero right-hand side for (2). The scalar metric perturbations generated during inflation have a size that gives density fluctations with 
$\delta \rho / \rho \sim 10^{-5}$. So it is interesting to speculate that a correct theory might realize the relation that we see numerically,

$$
\frac{n_{b}}{n_{\gamma}} \sim\left(\frac{\delta \rho}{\rho}\right)^{2} .
$$

Whether we succeed in realizing this intuition, we leave for the reader to judge.

We believe that our analysis is also interesting because the Sakharov conditions are satisfied in this scenario in an unusual way. Lepton number is of course violated through (2). CP violation and out-of-equilibrium result from the nonzero classical value of the axion field. Before inflation, the complex modulus field varies from point to point in both modulus and phase. Inflation blows up a small region in this field to a size much greater than that of the visible universe. In this region, the modulus field is approximately constant and has a randomly chosen, fixed phase. This value then rolls slowly toward the minimum of its potential. In this process, we have out-ofequilibrium dynamics and, if the phase is nonzero, a CP asymmetry. We claim that no explicit CP violation is needed in the equations of motion. The $\mathrm{CP}$-odd field $\phi$ could have zero expectation value today and need have no relation to the $\mathrm{CP}$ violation observed in particle physics.

\section{Gravity wave evolution}

Now we would like to quantitatively estimate the lepton number produced in inflation [11]. To begin, we must compute the production of gravitational waves in inflation under the influence of the coupling (4). The general form of metric perturbations about an FRW universe can be parametrized as

$$
d s^{2}=-(1+2 \varphi) d t^{2}+w_{i} d t d x^{i}+a^{2}(t)\left[\left((1+2 \psi) \delta_{i j}+h_{i j}\right) d x^{i} d x^{j}\right]
$$

where $\varphi, \psi, w_{i}$ and $h_{i j}$ respectively parametrize the scalar, vector, and tensor fluctuations of the metric. It is straightforward to show that the scalar and vector pertubations do not contribute to $R \tilde{R}$, and so we ignore these fluctuations in the following discussion. We can also fix a gauge so that the tensor fluctuation is parametrized by the two physical transverse traceless elements of $h_{i j}$. For gravity waves moving in the $z$ direction, we write

$$
d s^{2}=-d t^{2}+a^{2}(t)\left[\left(1-h_{+}\right) d x^{2}+\left(1+h_{+}\right) d y^{2}+2 h_{\times} d x d y+d z^{2}\right],
$$

where $a(t)=e^{H t}$ during inflation and $h_{+}, h_{\times}$are functions of $t, z$. To see the CP violation more explicitly, it is convenient to use a helicity basis

$$
h_{L}=\frac{1}{\sqrt{2}}\left(h_{+}-i h_{\times}\right), \quad h_{R}=\frac{1}{\sqrt{2}}\left(h_{+}+i h_{\times}\right) .
$$


Here $h_{L}$ and $h_{R}$ are complex conjugate scalar fields. To be very explicit, the negative frequency part of $h_{L}$ is the conjugate of the positive frequency part of $h_{R}$, and both are built from wavefunctions for left-handed gravitons.

The contribution of tensor perturbations to $R \tilde{R}$, up to second order in $h_{L}$ and $h_{R}$, is

$$
\begin{gathered}
R \tilde{R}=\frac{4 i}{a^{3}}\left[\left(\frac{\partial^{2}}{\partial z^{2}} h_{R} \frac{\partial^{2}}{\partial t \partial z} h_{L}-\frac{\partial^{2}}{\partial z^{2}} h_{L} \frac{\partial^{2}}{\partial t \partial z} h_{R}\right)+a^{2}\left(\frac{\partial^{2}}{\partial t^{2}} h_{R} \frac{\partial^{2}}{\partial t \partial z} h_{L}-\frac{\partial^{2}}{\partial t^{2}} h_{L} \frac{\partial^{2}}{\partial t \partial z} h_{R}\right)\right. \\
\left.+\frac{1}{2} \frac{\partial}{\partial t} a^{2}\left(\frac{\partial}{\partial t} h_{R} \frac{\partial^{2}}{\partial t \partial z} h_{L}-\frac{\partial}{\partial t} h_{L} \frac{\partial^{2}}{\partial t \partial z} h_{R}\right)\right] .
\end{gathered}
$$

If $h_{L}$ and $h_{R}$ have the same dispersion relation, this expression vanishes. Thus, for $R \tilde{R}$ to be nonzero, we need a 'cosmological birefringence' during inflation. Such an effect is induced by the addition of (4) to the gravitational equations [10].

Specifically, by adding (4) to the Einstein action, inserting (8), and varying with respect to the metric fluctuations, we find the equations of motion

$$
\square h_{L}=-2 i \frac{\Theta}{a} \dot{h}_{L}^{\prime}, \quad \square h_{R}=+2 i \frac{\Theta}{a} \dot{h}_{R}^{\prime},
$$

where

$$
\Theta=\frac{4}{M_{\mathrm{Pl}}^{2}}\left(F^{\prime \prime} \dot{\phi}^{2}+2 H F^{\prime} \dot{\phi}\right)
$$

dots denote time dervatives, and primes denote differentiation of $F$ with respect to $\phi$. Note that (4) with a constant $\phi$ is a total divergence that cannot affect the equations of motion; thus, all terms in $\Theta$ involve derivatives of $\phi$. We have dropped terms with third-order derivatives of $h_{L}$ and $h_{R}$ and terms with $\ddot{\phi}$. In fact, it is also permissible to ignore the $F^{\prime \prime}$ term in (12), since in slow-roll inflation, $\dot{\phi} \ll M_{\mathrm{Pl}} H$ and each derivative on $F$ brings a dimensionful factor of order $1 / M_{\mathrm{Pl}}$. These evolution equations should be compared to those for evolution in flat space given by LWK [10]. The new term proportional to $H \dot{\phi}$ leads to a substantial enhancement in the size of $\Theta$. With this simplification, and the approximate form (5),

$$
\Theta=\sqrt{2 \epsilon} \mathcal{N}\left(H / M_{\mathrm{Pl}}\right)^{2} / 2 \pi^{2},
$$

where $\epsilon=\frac{1}{2}(\dot{\phi})^{2} /\left(H M_{\mathrm{Pl}}\right)^{2}$ is the slow-roll parameter of inflation [11].

Let us now focus on the evolution of $h_{L}$ and, more specifically, on its positive frequency component. It is convenient to introduce conformal time

$$
\eta=\frac{1}{H a}=\frac{1}{H} e^{-H t}
$$


(Note that conformal time $\eta$ runs in the opposite direction from $t$.) The evolution equation for $h_{L}$ becomes

$$
\frac{d^{2}}{d \eta^{2}} h_{L}-2 \frac{1}{\eta} \frac{d}{d \eta} h_{L}-\frac{d^{2}}{d z^{2}} h_{L}=-2 i \Theta \frac{d^{2}}{d \eta d z} h_{L}
$$

If we ignore $\Theta$ for the moment and let $h_{L} \sim e^{i k z}$, this becomes the equation of a spherical Bessel function:

$$
\frac{d^{2}}{d \eta^{2}} h_{L}-2 \frac{1}{\eta} \frac{d}{d \eta} h_{L}+k^{2} h_{L}=0
$$

for which the positive frequency solution is

$$
h_{L}^{+}(k, \eta)=e^{+i k(\eta+z)}(1-i k \eta)
$$

We now look for solutions to (15) with $h_{L} \sim e^{i k z}$. To do this, let

$$
h_{L}=e^{i k z} \cdot(-i k \eta) e^{k \Theta \eta} g(\eta)
$$

Then $g(\eta)$ satisfies the equation

$$
\frac{d^{2}}{d \eta^{2}} g+\left[k^{2}\left(1-\Theta^{2}\right)-\frac{2}{\eta^{2}}-\frac{2 k \Theta}{\eta}\right] g=0 \text {. }
$$

This is the equation of a Schrödinger particle with $\ell=1$ in a Coulomb potential. When $\Theta=0$, the Coulomb term vanishes and we find the spherical Bessel function (17). For $h_{L}$, the the small Coulomb term is repulsive; for $h_{R}$, with the opposite sign of the $\Theta$ term, the Coulomb potential is attractive. This leads to attentuation of $h_{L}$ and amplification of $h_{R}$ in the early universe. This is just the cosmological birefringence described by LWK [10].

It will turn out that the generation of the matter asymmetry is dominated by modes at short distances (sub-horizon modes) and at early times. This corresponds to the limit $k \eta \gg 1$. In this region, we can ignore the potential terms in (19) and take the solution to be approximately a plane wave. More explicitly,

$$
g(\eta)=\exp \left[i k\left(1-\Theta^{2}\right)^{1 / 2} \eta(1+\alpha(\eta))\right]
$$

where $\alpha(\eta) \sim \log \eta / \eta$. 


\section{Green's function}

We would like to apply the results of the previous section to compute the expectation value of $R \tilde{R}$ in the inflationary space-time. Our expression will be dominated by the quantum part of the gravity-wave evolution. For this regime, we can calculate the expectation value by contracting $h_{L}$ and $h_{R}$ in $R \tilde{R}$ using an appropriate Green's function.

Define

$$
G\left(x, t ; x^{\prime}, t^{\prime}\right)=\left\langle h_{L}(x, t) h_{R}\left(x^{\prime}, t^{\prime}\right)\right\rangle=\int \frac{d^{3} k}{(2 \pi)^{3}} e^{i k \cdot\left(x-x^{\prime}\right)} G_{k}\left(\eta, \eta^{\prime}\right)
$$

For $k$ parallel to $z$, the Fourier component $G_{k}$ satisfies (15) with a delta-function source

$$
\left[\frac{d^{2}}{d \eta^{2}}-2 \frac{1}{\eta} \frac{d}{d \eta}+k^{2}-2 k \Theta \frac{d}{d \eta}\right] G_{k}\left(\eta, \eta^{\prime}\right)=i \frac{(H \eta)^{2}}{M_{\mathrm{Pl}}^{2}} \delta\left(\eta-\eta^{\prime}\right) .
$$

For $\Theta=0$, the solution of this equation is

$$
G_{k 0}\left(\eta, \eta^{\prime}\right)=\left\{\begin{array}{ll}
\left(H^{2} / 2 k^{3} M_{\mathrm{Pl}}^{2}\right) h_{L}^{+}(k, \eta) h_{R}^{-}\left(-k, \eta^{\prime}\right) & \eta<\eta^{\prime} \\
\left(H^{2} / 2 k^{3} M_{\mathrm{Pl}}^{2}\right) h_{L}^{-}(k, \eta) h_{R}^{+}\left(-k, \eta^{\prime}\right) & \eta^{\prime}<\eta
\end{array},\right.
$$

where $h_{L}^{-}$is the complex conjugate of $(17)$, and $h_{R}^{+}, h_{R}^{-}$are the corresponding solutions of the $h_{R}$ equation. For $\Theta=0$, these solutions are the same as for $h_{L}$, but the structure of (23) will be preserved when we go to the case $\Theta \neq 0$. The leading effect of $\Theta$ is to introduce the exponential dependence from (18),

$$
G_{k}= \begin{cases}e^{-k \Theta \eta} G_{k 0} e^{+k \Theta \eta^{\prime}} & \eta<\eta^{\prime} \\ e^{-k \Theta \eta} G_{k 0} e^{+k \Theta \eta^{\prime}} & \eta^{\prime}<\eta\end{cases}
$$

The prefactor is modified in order $\Theta^{2}$, and the wavefunctions acquire additional corrections that are subleading for $k \eta \gg 1$. Neither of these effects will be important for our result.

The Green's function (24) can now be used to contract $h_{L}$ and $h_{R}$ to evaluate the quantum expectation value of $R \tilde{R}$. The result is

$$
\langle R \tilde{R}\rangle=\frac{16}{a} \int \frac{d^{3} k}{(2 \pi)^{3}} \frac{H^{2}}{2 k^{3} M_{\mathrm{Pl}}^{2}}(k \eta)^{2} \cdot k^{4} \Theta+\mathcal{O}\left(\Theta^{3}\right)
$$

where we pick up only the leading behavior for $k \eta \gg 1$.

We note again that our expression for $\langle R \tilde{R}\rangle$ is nonzero because of the effect of inflation in producing a CP asymmetry out of equilibrium. The original quantum 
state for the inflaton might have had nonzero amplitude for a range of values of $\phi$ and might even have been CP-invariant. However, inflation collapses the wavefunction onto a particular value of $\phi$ that is caught up in the local expansion of the universe. This value gives us a classical background that is CP-asymmetric.

\section{$5 \quad$ Lepton asymmetry}

Now we are ready to evalute the lepton density that arises through (2). Inserting (25) into (2) and integrating over the time period of inflation, we find for the net lepton number density

$$
n=\int_{0}^{H^{-1}} d \eta \int \frac{d^{3} k}{(2 \pi)^{3}} \frac{1}{16 \pi^{2}} \frac{8 H^{2} k^{3} \eta^{2} \Theta}{M_{\mathrm{Pl}}^{2}} .
$$

The integral over $k$ runs over all of momentum space, up to the scale $\mu$ at which our effective Lagrangian description breaks down. The dominant effect comes not from the usual modes outside the horizon at the end of inflation (super-horizon modes), $k / H<1$, but rather from very short distances compared to these scales. The integral over $\eta$ is dominated at large values of $\eta$, early times. The integral represents a compromise between two effects of inflation, first, to blow up distances and thus carry us to smaller physical momenta and, second, to dilute the generated lepton number through expansion. It is now clear that the dominant contribution to the right-hand side comes from $k \eta \gg 1$, as we had anticipated. Performing the integrals, we find

$$
n=\frac{1}{72 \pi^{4}}\left(\frac{H}{M_{\mathrm{Pl}}}\right)^{2} \Theta H^{3}\left(\frac{\mu}{H}\right)^{6} .
$$

We might interpret this result physically in the following way. The factor $\left(H / M_{\mathrm{Pl}}\right)^{2}$ is the usual magnitude of the gravity wave power spectrum. The factor $\Theta$ gives the magnitude of effective $\mathrm{CP}$ violation. The factor $H^{3}$ is the inverse horizon size at inflation; this gives the density $n$ appropriate units. Finally, the factor $(\mu / H)^{6}$ gives the enhancement over one's first guess due to our use of strongly quantum, short

distance fluctuations to generate $R \tilde{R}$, rather than the super-horizon modes which effectively behave classically.

To understand the significance of this estimate, we should compare it to the entropy density of the universe just after reheating, assuming that the energy of the inflationary phase has been converted to the heat of a gas of massless particles. To estimate this, assume very naively that reheating is instantaneous. Then reheating converts an energy density $\rho=3 H^{2} M_{\mathrm{Pl}}^{2}$ to radiation with $\rho=\pi^{2} g_{*} T^{4} / 30$ and $s=2 \pi^{2} g_{*} T^{3} / 45$, where $g_{*}$ is the efffective number of massless degrees of freedom. 
This gives $s=2.3 g^{1 / 4}\left(H M_{\mathrm{Pl}}\right)^{3 / 2}$. With this value,

$$
\frac{n}{s}=6 \times 10^{-5} g^{-1 / 4}\left(\frac{H}{M_{\mathrm{Pl}}}\right)^{7 / 2} \Theta\left(\frac{\mu}{H}\right)^{6} .
$$

If we are less naive, we might follow the dilution of $n$ and $\rho$ with the expansion of the universe to the end of reheating. The final result is the same. Assuming that there have been no large increases in the entropy of the universe since the end of reheating, (28) can be compared directly to the present value of $n / s$ inferred from (1). For this one should note that the ratio of the present baryon number to the lepton number originally generated in leptogenesis is approximately $n_{B} / n=4 / 11$ [5]; then (1) implies $n / s=2.4 \times 10^{-10}$.

Inserting the estimate for $\Theta$ given in (13) and setting $g_{*} \sim 100$, we find

$$
\frac{n}{s} \sim 1 \times 10^{-6} \cdot \sqrt{\epsilon} \mathcal{N}\left(\frac{H}{M_{\mathrm{Pl}}}\right)^{11 / 2}\left(\frac{\mu}{H}\right)^{6} .
$$

The final result has $n / s \propto H^{-1 / 2}$, corresponding to $n \propto H^{1}$. Ignoring for the moment the dependence on $\mu$, the various factors accumulate to a very strong dependence on $\left(H / M_{\mathrm{Pl}}\right)$. Unfortunately, the ratio $\left(H / M_{\mathrm{Pl}}\right)$ is limited in simple slow-roll inflation from the relation $\delta \rho / \rho \sim\left(H / M_{\mathrm{Pl}}\right) / \sqrt{\epsilon} \sim 10^{-5}$. The WMAP results give a more precise version of this bound for the case of single-field inflation: $H / M_{\mathrm{Pl}}<1 \times 10^{-4}$ [12]. To make an estimate, put $\mu \sim H \sim 2 \times 10^{14} \mathrm{GeV}$; this yields

$$
n / s<10^{-28} \cdot \sqrt{\epsilon} \mathcal{N}
$$

a very small result for any reasonable choice of $\mathcal{N}$.

\section{Improvement of the lepton asymmetry}

The estimate (30) is rather unsatisfactory, but in several places we have used naive or pessimistic values of parameters. We should now ask by what factor (30) could reasonably be enhanced. Looking back to (28), the prefactor and the factor $\left(H / M_{\mathrm{Pl}}\right)^{7 / 2}$ are intrinsic to our mechanism. The remaining factors, however, are negotiable.

In the estimates above, we assumed that the mass scale from the kinetic term for the modulus is the Planck or string scale. If this mass scale is set at a lower mass $\mathcal{F}$, $\Theta$ can be larger, scaling as

$$
\Theta \sim \sqrt{2 \epsilon} \mathcal{N}\left(H / M_{\mathrm{Pl}}\right)^{2}\left(M_{\mathrm{Pl}} / \mathcal{F}\right) / 2 \pi^{2} .
$$


In principle, $\mathcal{N}$ can be a large dimensionless number, though values greater than 100 are probably difficult to accomodate with string theory. In the estimate (30), $\Theta$ is of the order of $10^{-9}$. It is straightforward to make $\Theta$ larger by three to four orders of magnitude.

Another possible source of enhancement is already apparent in (28). We can obtain a much larger value of $n / s$ by raising the value of $\mu$ at which our effective Lagrangian is valid. For example, taking $\mathcal{F} \sim \mu$ and setting $\mathcal{N} \sim 100, \epsilon \sim 10^{-2}$ and $H / M_{\mathrm{Pl}} \sim 10^{-4}$ we find

$$
\frac{n}{s} \sim 10^{-23} \cdot\left(\frac{\mu}{H}\right)^{5}
$$

Then we recover the observed value of $n / s$ for $\mu \sim 10^{17} \mathrm{GeV}$. This scale is somewhat larger than the conventional scale of supersymmetric grand unification, but perhaps these scales could be tied together in a specific model. Alternatively, it is consistent with our formulae to make $\mu$ and also $H$ lower. We could have $H$ as low as $1 \mathrm{TeV}$ with $\mu=8 \times 10^{15} \mathrm{GeV}$.

In summary, we have presented a new mechanism for the production of the cosmic baryon asymmetry. This mechanism relies on the axial vector anomaly to violate fermion number and on the initial state of inflation to both for $\mathrm{CP}$ violation and for out-of-equilibrium dynamics. These are very minimal ingredients that might be found in a wide variety of models of physics at short distances. The simple version of this model that we have analyzed here generates an acceptable values for the matter asymmetry only if we can make use of gravitational fluctuations somewhat above the usual grand unification scale. It is interesting to ask whether the conditions we have found can be embedded in a grand unification model in a natural way.

\section{ACKNOWLEDGEMENTS}

We thank our colleagues at SLAC and Stanford, particularly Yasaman Farzan, Shamit Kachru, Liam McAllister, Eva Silverstein and Scott Thomas, and also Niayesh Afshordi and William Unruh for fruitful discussions. We are grateful to Robert Brandenberger and Lee Smolin for several useful conversations and, most especially, for his encouragement during the course of this project. The work of MMSh-J is supported in part by the US National Science Foundation under grant PHY-9870115 and by funds from the Stanford Institute for Theoretical Physics. The work of SHSA and MEP is supported by the US Department of Energy under grant DE-AC03-76SF00515. 


\section{References}

[1] D. N. Spergel et al., Astrophys. J. Suppl. 148 (2003) 175, [arXiv:astro$\mathrm{ph} / 0302209]$.

[2] P. Huet and E. Sather, Phys. Rev. D 51, 379 (1995) [arXiv:hep-ph/9404302].

[3] For a recent review, see A. Riotto and M. Trodden, Ann. Rev. Nucl. Part. Sci. 49, 35 (1999) [arXiv:hep-ph/9901362].

[4] A. D. Sakharov, Pisma Zh. Eksp. Teor. Fiz. 5, 32 (1967) [JETP Lett. 5, 24 (1967)].

[5] V. A. Kuzmin, V. A. Rubakov and M. E. Shaposhnikov, Phys. Lett. B 155, 36 (1985); S. Y. Khlebnikov and M. E. Shaposhnikov, Nucl. Phys. B 308 (1988) 885.

[6] M. Fukugita and T. Yanagida, Phys. Lett. B 174, 45 (1986).

[7] M. Carena, M. Quiros, M. Seco and C. E. M. Wagner, Nucl. Phys. B 650, 24 (2003) [arXiv:hep-ph/0208043].

[8] L. Alvarez-Gaume and E. Witten, Nucl. Phys. B 234, 269 (1984).

[9] M. B. Green and J. H. Schwarz, Phys. Lett. B 149, 117 (1984).

[10] A. Lue, L. M. Wang and M. Kamionkowski, Phys. Rev. Lett. 83, 1506 (1999) [arXiv:astro-ph/9812088].

[11] Our conventions for inflationary cosmology follow A. R. Liddle and D. H. Lyth, Cosmological Inflation and Large-Scale Structure (Cambridge University Press, 2000). In particular, note that we use the reduced Planck mass $M_{\mathrm{Pl}}=2.44 \times 10^{18}$ $\mathrm{GeV}$.

[12] H.V. Peiris et al., Astrophys. J. Suppl. 148 (2003) 213, [arXiv:astro-ph/0302225]. 